\title{
Jenseits der rhetorischen Modernisierung? Geschlechterwissen zwischen Essenzialismus und Konstruktivismus
}

\author{
Julia Wustmann
}

\subsection{Die Bedeutsamkeit des Geschlechterwissens pädagogischer Fachkräfte in frühkindlichen Kontexten}

Neben der Familie als primärer Sozialisationsinstanz bilden Kindertageseinrichtungen den ,wesentlichste[n] Sozialisationsraum“ für Kinder (Rohrmann und Wanzeck-Sielert 2018, S. 62). Denn in keinem anderen frühkindlichen, institutionellen Setting greifen die Betreuung, die Bildung sowie die Erziehung von Kindern derart intensiv ineinander, wie es bei Kindertageseinrichtungen der Fall ist (Rohrmann und Wanzeck-Sielert 2018, S. 62; Rossbach et al. 2012). Als eine zentrale Institution der frühkindlichen Sozialisation sind Kindertageseinrichtungen ein Bestandteil der gesellschaftlichen Ordnung und sie sind in der Folge auch von den Mustern und Regeln dieser Ordnung durchzogen (Rabe-Kleberg 2003, S. 26). Dieser Umstand gilt auch für Strukturen der ,gesellschaftlichen Geschlechterordnung" (Rabe-Kleberg 2003, S. 26). Denn innerhalb von Tageseinrichtungen erfahren, erlernen und erproben Kinder auch Vorstellungen zu Geschlechterverhältnissen, -typisierungen oder auch -gerechtigkeit. Daher werden Kindertageseinrichtungen auch als „gendered institution“, vergeschlechtlichte Institutionen, bezeichnet (Rabe-Kleberg 2003, S. 26; Rohrmann und Wanzeck-Sielert 2018).

Eine bedeutsame Rolle kommt in diesem Kontext dem Wissen zu, das die in Kindertageseinrichtungen tätigen pädagogischen Fachkräfte zum Thema

\footnotetext{
J. Wustmann ( $\varangle)$

Fakultät 17 Sozialwissenschaften, Technische Universität Dortmund, Dortmund, Deutschland

E-Mail: julia.wustmann@tu-dortmund.de
} 
Geschlecht haben. Denn sie sind nicht nur qua ihrer beruflichen Rolle dazu angehalten, Kindern explizit Wissensinhalte zu vermitteln, sondern sie nehmen in den Bildungsprozessen von Kindern implizit auch eine Vorbildfunktion ein (Fried et al. 2012, S. 52 f.). Pädagogische Fachkräfte verfügen neben einem alltagsweltlichen, ,subjektive[n] ,Geschlechter-Wissen“ auch über ein „objektivierte[s]" Geschlechterwissen (Dölling 2005, S. 50), worunter unterschiedliche Wissensformen gefasst werden,

[...] die in einer Gesellschaft jeweils als kollektiver Wissensvorrat über den Geschlechterunterschied, die Begründungen seiner ,Selbstverständlichkeit“ und Evidenz, über die (vor-)herrschenden normativen Vorstellungen von den ,richtigen“ Beziehungen und Arbeitsteilungen zwischen Frauen und Männer existieren [...]. (Dölling 2005, S. 50)

Das in der beruflichen Ausbildung verwendete Lehrmaterial stellt einen solchen kollektiv geteilten, objektivierten Wissensvorrat dar. Welches Wissen zu Geschlecht hier vermittelt wird, ist insoweit von Bedeutung, als dass das in Form des Lehrmaterials objektivierte, fachspezifische Geschlechterwissen für angehende Fachkräfte als eine Orientierung dient, an dem das Handeln in der beruflichen Praxis ausgerichtet werden soll. So beeinflusst das vermittelte Geschlechterwissen des Lehrmaterials etwa die Art und Weise, wie pädagogische Fachkräfte - sowohl gegenüber Kindern als auch im pädagogischen Team - Fragen nach der Bedeutung von Geschlechterunterschieden und -gemeinsamkeiten beantworten, wie sie auf geschlechterdifferenzierendes Handeln von Kindern und Kolleginnen und Kollegen (re-)agieren und wie sie eine gleichberechtigte Erziehung von Kindern sowie ein gleichberechtigtes Arbeiten im Team gestalten. Aufgrund der Tragweite des in der Ausbildung von pädagogischen Fachkräften vermittelten objektivierten Geschlechterwissens wurde im Forschungsprojekt das in der deutschsprachigen Schweiz verwendete und aufgrund der Anpassung des Bildungsplans 2011 aktualisierte, überarbeitete und neu aufgelegte Ausbildungshandbuch Fachfrau/Fachmann Betreuung untersucht.

Die theoretische Konzeption des Forschungsvorhabens folgte der Annahme der sozialen Konstruiertheit von Geschlecht. In einer solchen Perspektive wird zum einem der Umstand berücksichtigt, dass soziale Wirklichkeit zweigeschlechtlich (Frau/Mann, Mädchen/Junge, weiblich/männlich) strukturiert ist. Zum zweiten und daran anschließend wird die Annahme vertreten, dass die Geschlechterdifferenzierung ,eine permanent stattfindende soziale Praxis“ ist (Hirschauer 1996, S. 242, Hervorhebungen im Original), innerhalb derer die Geschlechterunterscheidung überhaupt erst hergestellt wird. Geschlecht wird also nicht als eine 
essenzielle Eigenschaft eines Individuums, sondern als ein in Interaktionen über das Handeln produziertes, soziales Phänomen verstanden - eine Annahme, die begrifflich im Konzept des doing gender gefasst wird (West und Zimmerman 1987; Gildemeister und Wetterer 1992; Gildemeister 2008).

Das Geschlechterwissen, das es innerhalb des dargestellten Forschungsvorhabens zu rekonstruieren galt, ist ,für die Praxis des ,doing gender “ unverzichtbar“, umfasst es doch „ein vielfältiges Repertoire von Praktiken der Wissensgenerierung, die im ,doing "in Sekundenbruchteilen ineinander greifen" (Wetterer 2008a, S. 23). Aus dieser konstruktivistischen Sichtweise heraus rückten in der empirischen Rekonstruktion des im Ausbildungsmaterial vermittelten Geschlechterwissens entsprechend nicht Geschlechterunterschiede, sondern Prozesse der Geschlechterunterscheidung in den Fokus der analytischen Aufmerksamkeit.

Theoretisch würde es jedoch zu kurz greifen, von einer Omnipräsenz der bipolaren Geschlechterdifferenz auf eine Omnirelevanz der Geschlechterunterscheidung zu schließen (Heintz und Nadai 1998; Hirschauer 1994). Zwar teilen wir in einer sozialen Interaktion in der Regel unserem Gegenüber bereits über Gestiken, Frisuren, Kleidungen etc. unser Geschlecht mit (Omnipräsenz), doch ergibt sich daraus nicht notwendigerweise, dass unser Geschlecht in einer sozialen Interaktion vorrangig von Bedeutung ist (Omnirelevanz):

Die Geschlechtlichkeit von Personen mag zwar als Hintergrunderwartung immer präsent sein, aber nicht in jedem Kontext wird das Geschlecht zu einer sozialen Ordnungskategorie. Es gibt Situationen, in denen Geschlechtszugehörigkeit durch andere soziale Merkmale überdeckt oder auch gezielt neutralisiert wird. (Heintz und Nadai 1998, S. 82)

Theoretisch wird der Umstand, dass Geschlecht in sozialen Interaktionen nicht immer von vorrangiger Relevanz ist, auch wenn dessen Präsenz allgegenwärtig ist, im Konzept des undoing gender gefasst. Die Auffassung des situativ-prozessualen undoing gender varriert jedoch innerhalb der Sozialwissenschaften (vgl. Kap. 2) und reicht von der Unterbrechung bzw. Unterlassung der Geschlechterunterscheidung (Hirschauer 2001) über den Widerstand gegen die Praktiken der Geschlechterunterscheidung (Butler 2004), das Verändern von Geschlechternormen (West und Zimmermann 2009) bis hin zur Betonung der Geschlechtergleichheit (Deutsch 2007), statt der Betonung der Geschlechterdifferenz (Vogt et al. 2015, S. 230 f.).

Entsprechend dieser operationalisierenden Überlegungen zum undoing gender wurde der Fokus der Analyse des Ausbildungsmaterials nicht nur auf die Reproduktion der Geschlechterunterscheidung gelegt (doing gender), sondern auch 
darauf, an welchen Stellen und auf welche Weise die Geschlechterunterscheidung unterlassen oder gestört, von etablierten Geschlechternormen abgewichen oder aber Geschlechtergleichheit (im Gegensatz zu Geschlechterdifferenz) hervorgehoben wurde (undoing gender).

Vor dem Hintergrund der konzeptionellen Entwicklungen und Überlegungen zum (un)doing gender wurden für die Analyse des Ausbildungsmaterials von Fachfrauen/Fachmänner Kinderbetreuung schließlich folgende Forschungsfragen abgeleitet:

a) Welches Geschlechterwissen wird im Ausbildungsmaterial $\mathrm{zu}$ Fachfrau/Fachmann Kinderbetreuung vermittelt?

b) Welche geschlechtsspezifischen Aspekte werden im Lehrmaterial thematisiert und auf welche Weise werden diese verhandelt?

c) Wie werden Fachfrauen/Fachmänner Betreuung auf den Umgang mit Geschlechterthematiken in der beruflichen Praxis im Rahmen der Ausbildung vorbereitet?

Zur Beantwortung dieser Forschungsfragen wird im Folgenden zunächst die konzeptionelle Fassung des zu analysierenden Ausbildungsmaterials und des darin vermittelten Geschlechterwissens erläutert. In einem darauffolgenden Schritt wird die methodische Vorgehensweise zur Analyse des Datenmaterials plausibilisiert, bevor exemplarische Interpretationen zur Rekonstruktion des materialimmanenten Geschlechterwissens vorgestellt werden. Abschließend wird diskutiert, wie die Ergebnisse theoretisch gefasst werden können und welche Auswirkungen sich aus dem Geschlechterwissen, das im Ausbildungsmaterial vermittelt wird, für die Ausbildung von Fachfrauen/Fachmännern Betreuung ergeben.

\subsection{Zur theoretischen Konzeptualisierung von Geschlechterwissen in Lehrmaterialien}

In einem ersten Schritt soll nun erkundet werden, als was für eine Datenmaterialsorte das zu untersuchende Ausbildungsmaterial theoretisch gefasst werden kann, um daran anschließend zu klären, was sich aus dieser Erkenntnis konzeptionell für die Erforschung des im Ausbildungsmaterial vermittelten Geschlechterwissens ableiten lässt. Das zu untersuchende Ausbildungsmaterial lässt sich, verstanden als eine Aneinanderreihung schriftlich fixierter Äußerungen, zunächst einmal der Datenmaterialsorte des Dokuments zuordnen (Reh 1995, S. 204). Dokumente werden im Allgemeinen zur Archivierung von Materialien verwendet, fungieren 
im Speziellen aber auch „als vorbildliche Mustergabe für Lern- und Lehrstücke“ (Kraimer 2009, S. 2). Das zu untersuchende Ausbildungsmaterial kann an diese Ausführungen anschließend als ein Dokument im Sinn eines vorbildlichen Lehrmittels verstanden werden, da das Material als bedeutsam angesehene Inhalte für die Ausbildung pädagogischer Fachkräfte in schriftlich fixierter Form umfasst.

Die wissenschaftliche Forschungslandschaft zu Lehrmitteln ist durch Analysen zur spezifischen Lehrmittelform des Schulbuchs dominiert. Auch wenn es sich beim vorliegenden Datenmaterial nicht um ein Schulbuch handelt, sondern um ein Ausbildungsmaterial, so fallen beide Dokumentenarten unter die Oberkategorie des Lehrmittels und teilen den grundlegenden Zweck der Vermittlung von Wissen in einem (Aus-)Bildungskontext. Daher verspricht ein Einblick in die Charakteristika des Schulbuchs auch Aufschluss über kennzeichnende Merkmale des Ausbildungsmaterials für Fachfrau/Fachmann Kinderbetreuung.

Das Schulbuch wird als ein Medium gefasst, das - eingesetzt in Unterrichtssituationen - fachwissenschaftliche und fachdidaktische Forschungserkenntnisse vermittelt. Das Schulbuch ist also ein für Unterrichtssituationen konzipiertes Lehr-, Lern- und Arbeitsmittel, das den gegenwärtigen, forschungsbasierten Erkenntnisstand eines Fachbereichs versammelt (Sandfuchs 2010, S. 19). Das Schulbuch kann, hinsichtlich seiner spezifischen Textart, zwischen dem Typus des Sachbuchs und dem des wissenschaftlichen Fachbuchs verortet werden (Wiater 2003, S. 12). Darüber hinaus erklärt sich ein Schulbuch

\begin{abstract}
„immer auch aus politischen und pädagogischen Setzungen. Das zeigt sich an den Auswahlgesichtspunkten bei den präsentierten Lerninhalten, an den Schwerpunktsetzungen und den im Schulbuchvergleich erkennbar werdenden Akzentuierungen und Abgrenzungen. Infolgedessen muss das Schulbuch theoretisch als Politikum, Informatorium und Paedagogicum betrachtet werden [...], eingebettet in einen politischen, pädagogisch-didaktischen und gesellschaftlich-ökonomischen Kontext" (Wiater 2003, S. 12).
\end{abstract}

Das Ausbildungsmaterial für Fachfrau/Fachmann Betreuung wird entsprechend dieser Ausführungen weiter spezifiziert und als ein in Dokumentenform vorliegendes Lehrmedium verstanden, das fachspezifisches Wissen der Pädagogik didaktisch vermitteln soll. Das Ausbildungsmaterial bildet jedoch das fachspezifische Wissen nicht in neutraler Weise ab, sondern produziert, bedingt durch die in der Konzeption des Materials getroffene Auswahl bestimmter Inhalte, durch die Einbeziehung und Auslassung von Forschungserkenntnissen und auch durch die Entscheidung für oder gegen die Aufnahme von Kritikpunkten, eine ganz eigene Form von Wissen (Lässig 2010). 
Dem Forschungsschwerpunkt folgend ist vor allem das im Ausbildungsmaterial vermittelte Geschlechterwissen von besonderem Interesse. Da der Sinn und Zweck von Lehrmitteln in der Vermittlung fachwissenschaftlicher Forschungserkenntnisse besteht, kann ein zu rekonstruierendes Geschlechterwissen des Ausbildungsmaterials als ein „systematisches Wissen über das Wissen“ gerahmt werden (Wetterer 2008a, S. 16). Denn bei einem solchen Lehrmittel zu Geschlecht handelt es sich um ein didaktisch aufbereitetes Wissen über wissenschaftliches Geschlechterwissen (Dölling 2005). Zu beachten ist entsprechend, dass das materialspezifische Geschlechterwissen mitunter auch pädagogischen und politischen Setzungen unterliegt, die es im stetigen Vergleich mit dem gegenwärtigen geschlechtersoziologischen Geschlechterwissens zu reflektieren gilt.

Der Beginn einer breiteren empirischen Auseinandersetzung mit Geschlechterwissen in Lehrmaterialien lässt sich für die deutschsprachige Forschung auf die 1970er- und 1980er-Jahre datieren (Barz 1982; Borries 1975; Elbracht und Mosler 1972; Glötzner 1982). Wie Annette Hunze (2003) in einer überblicksartigen Zusammenfassung der Ergebnisse von Schulbuchanalysen der 1970er-, 1980erund 1990er-Jahre aufzeigt, lassen sich diverse Wandlungsprozesse hinsichtlich der Darstellung von Geschlechterverhältnissen nachzeichnen. So ist die zahlenmäßige Repräsentanz von weiblichen Personen in Schulbüchern im Lauf der Jahr(zehnt)e deutlich angestiegen, auch wenn Frauen insgesamt statistisch immer noch unterrepräsentiert sind. Für die Darstellung von männlichen Personen wird eine Steigerung der Repräsentanz in bestimmten thematischen Bereichen (wie etwa der Familie) konstatiert (Hunze 2003, S. 59-79).

Trotz einiger Wandlungsprozesse in der Darstellung der Geschlechterverhältnisse seien nach Hunze (2003) die Darstellungen von Frauen und Männern jedoch immer noch durch Geschlechtsstereotype dominiert. Mit Blick auf die eingangs dargestellten Konzeptionen von doing und undoing gender lässt sich angesichts dieser Ergebnisse schlussfolgern, dass Schulbücher der 1970er-, 1980er- und 1990er-Jahre durch ein konsequentes doing gender charakterisiert sind, insoweit die Geschlechterunterscheidung in den Darstellungen auch trotz konstatierter Wandlungsprozesse stets neu produziert wird.

Die Ergebnisse aktuellerer Untersuchungen zu Geschlechterwissen in Schulbüchern zeigen, dass ungleiche Verhältnisse in der Repräsentation von männlichen und weiblichen Personen (weiterhin) bestehen, dass Geschlechterdifferenzierungen durchgehend Anwendung finden und dass Heteronormativität innerhalb der Darstellungen in Schulbüchern dominiert (Bauer 2007; Bittner 2015; Finsterwald und Ziegler 2007; Markom und Weinhäuptl 2007; Moser et al. 2013). Somit lässt sich auch für gegenwärtige Lehrmaterialien schlussfolgern, dass diese durch ein konsequentes doing gender geprägt sind, denn vorrangig wird in diesen die 
Geschlechterunterscheidung vielmehr betont (und somit neu produziert), als dass sie stattdessen etwa negiert, hinterfragt, unterlassen oder gestört wird.

Für die Analyse des Ausbildungsmaterials Fachfrau/Fachmann Betreuung lässt sich aus diesem grob skizzierten Forschungsüberblick nochmals eine besondere Aufmerksamkeit für Prozesse der (Re-)Produktion der Geschlechterunterscheidung (doing gender), aber auch eine erhöhte Sensibilität hinsichtlich der (Re-)Produktion von Geschlechterungleichheiten ableiten. Die im Vorangegangenen dargestellten Ergebnisse bisheriger Forschungsarbeiten zu Geschlechterwissen in Lehrmaterialien zeigen darüber hinaus auf, dass das Konzept des undoing gender in bisherigen Forschungen randständig bis gar nicht einbezogen wurde. Die im hier vorgestellten Forschungsansatz vorgenommene Erweiterung der analytischen Einstellung über die Hinzuziehung des undoing-gender-Konzepts scheint daher besonders erkenntnisversprechend.

\subsection{Die sozialwissenschaftliche Hermeneutik als methodisches Verfahren zur Rekonstruktion von Geschlechterwissen}

Der Prozess der sozialen Konstruktion der Geschlechterunterscheidung (doing gender) verläuft aufgrund der bestehenden zweigeschlechtlichen Strukturiertheit der Gesellschaft ,unsichtbar und tritt uns im Ergebnis als so hochgradig selbstverständlich entgegen, dass schon die Frage nach dem Herstellungsmodus i. d. R. Irritationen auslöst“" (Gildemeister 2008, S. 172). Für die Rekonstruktion sozialer Konstruktionsprozesse (doing gender) und den ihnen zugrunde liegenden Wissensvorräten (Geschlechterwissen) braucht es also entsprechend ein methodisches Auswertungsverfahren, das die Herausarbeitung latenter Sinnstrukturen ermöglicht.

Vor diesem Hintergrund wurde die sozialwissenschaftliche Hermeneutik nach Soeffner als methodisches Auswertungserfahren gewählt, denn diese beabsichtigt, ,etwas über das in den Dokumenten und in der Deutung enthaltene Wissen [zu] wissen, über seine Herkunft, Konstitution, Wirksamkeit und über seine Alternativen“ (Soeffner 2004, S. 108). Erreicht wird dieses Vorhaben über das Explizieren des impliziten Wissens, wodurch, ,nicht nur auf eine Deutung des Gewussten, sondern auf die Konstitutionsregeln und Bedingungen des Wissens selbst abgezielt wird“ (Soeffner 2004, S. 108). Nach Soeffner geht es bei der sozialwissenschaftlichen Hermeneutik allerdings nicht darum herauszufinden, was bei Sinnkonstruktionen im Kopf einer Sprecherin oder eines Sprechers vorgeht. Ein solches „Inneres“ ist nach Soeffner stets ein „,schweigsames Inneres“, denn 
solange es nicht in Äußerungen nach außen dringt, solange kann es aus einer fremdverstehenden Perspektive heraus nicht interpretiert werden (Soeffner 2004, S. 84, 213). Verschriftlichungen in Dokumentenform, wie sie mit dem zu untersuchenden Ausbildungsmaterial vorliegen, bilden eine Variante, wie ein solches „schweigsames Inneres“ nach außen hin geäußert werden kann.

Die schriftliche Vertextung sozialer Wirklichkeit als Voraussetzung für die hermeneutische Interpretation bedingt jedoch eine ,permanente Textproduktion“, woraus sich notwendigerweise ableitet, dass ,das mühsame Geschäft der Interpretation und Textauslegung immer hinter der Menge der interpretierten Dokumente zurückbleiben muss" (Soeffner 2004, S. 157 f.). Daraus ergibt sich die Konsequenz, dass die sozialwissenschaftliche Hermeneutik durchweg exemplarisch vorgeht und an sich eine Fallanalyse ist (Soeffner 2004, S. 158). Das Vorgehen der sozialwissenschaftlichen Hermeneutik beabsichtigt folglich nicht, das gesamte vorliegende Datenmaterial zu bearbeiten. Vielmehr besteht das Ziel einer solchen exemplarisch vorgehenden Fallanalyse darin, die latenten Sinnstrukturen, die einem Dokument immanent sind, interpretativ-nachvollziehbar zu rekonstruieren.

\subsubsection{Auswahl und Rahmung des zu analysierenden Datenmaterials}

In Vorbereitung auf die Analyse wurde zunächst das über 3000 Seiten umfassende Datenmaterial gesichtet. Entsprechend des Forschungsfokus auf die Ausbildung zur Kinderbetreuung wurden zum einen das Material der „Allgemeinen Berufskunde", das in der Ausbildung aller pädagogischen Berufszweige Anwendung findet, und zum anderen das Material zur „Spezifischen Berufskunde, Fachrichtung Kinderbetreuung" ausgewählt. Die Sichtung potenziell zu analysierender Passagen innerhalb dieser Kapitel orientierte sich wiederum an den Forschungsfragen.

Hinsichtlich der ersten beiden Forschungsfragen nach dem im Material vermittelten Geschlechterwissen und der Verhandlung geschlechtsspezifischer Aspekte wurden Passagen ausgewählt, in denen dezidiert Themen mit Bezug zu Geschlecht behandelt wurden. Als zentrales Kapitel wurde diesbezüglich das Unterkapitel "Gender - Männer weinen nicht" aus der Allgemeinen Berufskunde in die Analyse einbezogen. Da die dritte Forschungsfrage darauf abzielte, zu rekonstruieren, wie den angehenden Fachfrauen/Fachmännern der zukünftige Umgang mit Geschlechterthematiken vermittelt wird, dieser Prozess aber nicht in Form von teilnehmender Beobachtung realisiert werden konnte, wurde das Datenmaterial auch auf Vermittlungsaspekte hin gesichtet. Diesbezüglich wurden die sogenannten Praxissituationen in die Analyse aufgenommen. Dabei handelte es sich um 
imaginierte, potenzielle Situationen und Beispiele aus der beruflichen Praxis, die thematisch von Bewältigungsvorschlägen zu alltäglichen Problemsituationen bis hin zu verschiedenen zu erfüllenden Aufgaben in Vorbereitung auf die berufliche Praxis reichten.

Da sich die ausgewählten Kapitel und Passagen hinsichtlich der Art und Weise, wie Geschlechterwissens vermittelt wird, auffallend unterscheiden (theoretisches Wissen über Geschlecht vs. über Praxisbezüge vermitteltes Wissen über Geschlecht), gilt es, die ausgewählten Materialstellen nochmals theoretisch zu differenzieren. Den Hintergrund dazu bildet das Konzept der ,rhetorischen Modernisierung“ von Wetterer (2003). Sie argumentiert, dass gegenwärtige Geschlechterverhältnisses ,durch Widersprüche, Brüche und Ungleichzeitigkeiten gekennzeichnet" seien (Wetterer 2003, S. 288). Sie führt die Widersprüchlichkeiten darauf zurück, ,dass sich Kultur- und Strukturzusammenhang gegeneinander verschoben haben“, wodurch eine Diskrepanz zwischen dem diskursiven Wissen (Rhetorik) und dem alltagsweltlichen Handeln (Praxis) entstanden sei (Wetterer 2003, S. 289).

Die von Wetterer vorgenommene Dimensionalisierung in Diskurs und Praxis lässt sich sinngemäss auf die verschiedenen Arten vermittelten Geschlechterwissens im Ausbildungsmaterial übertragen: Das im Kapitel „Gender - Männer weinen nicht" vermittelte, didaktisch aufbereitete Wissen über wissenschaftliches Geschlechterwissen lässt sich auf einer diskursiven Ebene verorten. Das in den Praxissituationen vermittelte Wissen zu Geschlecht lässt sich dagegen auf einer handlungspraktischen Ebene verorten, da in diesen Beispielen die (imaginierte) Alltagspraxis der Kinderbetreuung behandelt wird. Entsprechend dieser Dimensionalisierung gilt es in der Analyse auch das Verhältnis dieser beiden Dimensionen von Geschlechterwissen zueinander zu bestimmen.

\subsubsection{Analytische Vorgehensweise der sozialwissenschaftlichen Hermeneutik}

Die ausgewählten Passagen und Ausschnitte des zu untersuchenden Ausbildungsmaterials wurden im Sinn Soeffners als „Protokoll[e] irreversibler Interaktionsund Interpretationssequenzen gefasst“" (Soeffner 2004, S. 81). Damit ist gemeint, dass das Datenmaterial nicht nur als eine bloße Aneinanderreihung von Einzeläußerungen verhandelt wird, sondern dass die Einzeläußerungen immer aufeinander und darüber hinaus auch auf einen abstrakteren Handlungsrahmen Bezug nehmen. Dies gilt nach Soeffner selbst im Sonderfall des Monologs (Soeffner 2004, S. 79), als der das Ausbildungsmaterial im weitesten Sinn gefasst werden kann. 
Denn mit der Verschriftlichung des Ausbildungsmaterials wenden sich die Verfassenden monologisierend an die Lesenden, die interaktiv nicht darauf reagieren können.

Die Interpretation erfolgte sequenzanalytisch (vgl. Soeffner 2004, S. 83), was bedeutete, dass bei der Interpretation eines Interakts nicht auf nachfolgende Interakte vorgegriffen wurde, um zu verhindern, dass der Deutungshorizont frühzeitig verengt wird. Daran schloss sich wiederum an, dass während der Interpretation erarbeitete „Grundannahmen [...] als Handlungs- und Sinnhorizont des Folgeinteraktes so lange weiter [galten], bis sie durch den Text selbst expressis verbis aufgehoben oder widerlegt" wurden (Soeffner 2004, S. 85, Hervorhebungen im Original). Umgesetzt wurde dieses Vorhaben, indem zunächst Wort für Wort, später Satz für Satz interpretiert wurde, ohne die nachfolgenden Textstellen einzubeziehen.

Mit diesem sequenzanalytischen Vorgehen wurde in einem ersten Schritt die „egologisch-monothetische Perspektive“ rekonstruiert, womit Soeffner die subjektive Intentionalität des Sprechers bzw. der Sprecherin (bzw., im Fall des Ausbildungsmaterial, die der Verfassenden) umschreibt (Soeffner 2004, S. 83). In einem zweiten Schritt wurde die rekonstruierte "egologisch-monothetische Perspektive“ mit möglichst vielen objektiv denkbaren Les- und Deutungsarten konfrontiert, um eine ,polythetisch[e] Perspektivenneutralität“" zu erreichen (Soeffner 2004, S. 83). Während dieses Schritts der Interpretation des Ausbildungsmaterials wurden sukzessiv Thesen generiert, die im Fortgang der Interpretation entweder über weitere Interakte des Datenmaterials bestätigt und weitergetragen oder aber widerlegt wurden.

Dadurch sollte zum einen dem „Verifikationsgebot wissenschaftlicher Interpretation" (Soeffner 2004, S. 85) Rechnung getragen werden und zum anderen sollte eine intersubjektive Nachvollziehbarkeit gewährleistet werden. In einem dritten Schritt wurde einerseits beschrieben, welche der möglichen Deutungen der Verfasser bzw. die Verfasserin gewählt hat, und andererseits rekonstruiert, warum genau diese und keine andere Wahl der Deutung getroffen wurde. Ergänzt wurde dieser Schritt durch eine diskursive Verortung der Ergebnisse, indem datenmaterialexterne Fachliteratur der gegenwärtigen Geschlechtersoziologie hinzugezogen wurde. 


\section{4 "Same same but different" - Rekonstruktion des Geschlechterwissens im Ausbildungsmaterial Fachfrau/Fachmann Betreuung}

Im Folgenden werden die zentralen Ergebnisse der sozialwissenschaftlich hermeneutischen Analyse des im Ausbildungsmaterials vermittelten Geschlechterwissens dargestellt. Es wird zunächst ein Einblick in Rekonstruktion des diskursiven Geschlechterwissens gegeben und anschließend die analytische Rekonstruktion des handlungspraktischen Geschlechterwissens exemplarisch dargestellt.

\subsubsection{Geschlecht $\neq$ gender - das diskursive Geschlechterwissen zwischen Konstruktivismus und Essenzialismus}

Einen ersten, wohlgleich aber sehr grundlegenden Einblick in das diskursive Geschlechterwissen, das im Ausbildungsmaterial vermittelt wird, erhält man über die Definition einer begrifflichen Relation, die zu Beginn des Abschnitts „Gender - Männer weinen nicht“ ausgeführt wird. So werden die Termini Geschlecht und gender als zentrale Begriffe des Materials voneinander unterschieden und wie folgt in Relation zueinander definiert:

\footnotetext{
,Geschlecht ' bezieht sich auf die Unterscheidung in männliche und weibliche Lebewesen, die sich zum Zweck der Fortpflanzung miteinander vereinen. ,Geschlecht " ist also ein Fachbegriff, der primär in der Biologie verwendet wird. In sozialwissenschaftlichen Disziplinen wie der Soziologie und der Psychologie benutzt man aber eher den Begriff ,gender ${ }^{\text {, }}$, der sich nicht ausschließlich auf die biologische, sondern auf die sozio-kulturelle und psychologische Unterscheidung in das ,typisch Männliche“ und das ,typisch Weibliche‘ bezieht. (ABK, J51)
}

Die Begriffe Geschlecht und gender werden zunächst als fachspezifische Termini dargestellt, die in einem Sinnzusammenhang mit der Unterscheidung von Individuen in weiblich und männlich gebracht werden. Beide Termini werden darüber hinaus zueinander in Beziehung gesetzt, insoweit dem Begriff gender zugeschrieben wird, im Vergleich zum Begriff Geschlecht, semantisch nicht nur die biologische, sondern auch die die soziokulturelle und die psychologische Unterscheidung von Frauen und Männern zu beinhalten. Somit wird gender als ein erweiterter Geschlechtsbegriff konzipiert, der nicht nur eine, sondern mehrere Bedeutungsebenen umfasst. Die geschlechtsbezogene Unterscheidung, die dem Begriff Geschlecht zugeordnet wird, wird mit einem Zweck, also einer 
essenzialistischen Sinnhaftigkeit verbunden, die das (Fort-)Bestehen von Menschen tangiert: die Fortpflanzung. Dem Terminus gender wird dagegen keine solche Sinnhaftigkeit zugeschrieben, wodurch dieser im Vergleich weit weniger bedeutsam erscheint.

Darüber hinaus wirkt der Begriff gender über die gegebene Definition auch weitaus schwammiger, insoweit sich die Geschlechterunterscheidung, die mit ihm einhergeht, auf das Typische von Männlichkeit und Weiblichkeit bezieht. Wird etwas als typisch beschrieben, so sind damit in der Regel charakteristische Kennzeichen oder Merkmale von etwas gemeint. Es wird jedoch - anders als beim biologisch gefassten Begriff Geschlecht - nicht konkretisiert, was als typisch weiblich bzw. männlich gilt. Dies kann zum einen meinen, dass angenommen wird, das Typische von Männlichkeit und Weiblichkeit sei ein Allgemeingut und es bestehe daher keine Notwendigkeit zur Erklärung. Oder aber es wird davon ausgegangen, dass das Typische derart komplex ist, dass es nicht in einer vergleichsweise simplen (und sinnhaften) Art und Weise definiert werden kann.

Im weiteren Verlauf des Abschnitts wird die Unterschiedlichkeit der Termini Geschlecht und gender noch ausführlicher ausgeführt. So sei mit Geschlecht „die naturbedingte Unterscheidung: männlich/weiblich“ gemeint und die Bestimmung einer Person als Frau oder Mann erfolge durch „biologisch bestimmte körperliche Geschlechtsmerkmale“ (ABK, J52). Dass die Natur als Begründung für den Unterschied zwischen weiblich und männlich angeführt wird (,naturbedingt“), suggeriert, dass es sich bei dem Geschlechterunterschied um eine essenzielle Differenz handle. Denn Natur wird oftmals als Gegenbegriff zur Kultur verwendet und impliziert in dieser relationalen Bestimmung vor allem eine ursprüngliche, unabänderliche Gegebenheit. Diese essenzielle Annahme zur Geschlechterunterscheidung wird argumentativ mit der Körperlichkeit von Individuen verbunden, indem auf „körperliche Geschlechtsmerkmale“ als Marker für die Unterscheidung von Individuen in männlich und weiblich verwiesen wird. Der Körper wird auf diese Weise zu einem Anzeiger der Geschlechtszugehörigkeit erklärt, die wiederum als eine essenzielle Eigenschaft von Individuen definiert wird.

Gender sei hingegen „konstruiert“ (ABK, J52) und die angehenden Fachfrauen/Fachmänner Betreuung müssten sich darüber „,bewusst sein, dass gender nicht statisch ist, sondern dass es als soziales Gebilde ständig wieder neu ,produziert "“ wird (ABK, J59). Mit dem Hinweis auf die Konstruiertheit von gender wird impliziert, dass die soziokulturell und psychologisch fundierte Unterscheidung von Frauen und Männern ein Produkt eines Herstellungsprozesses sei. Diese Lesart wird nochmals durch den Terminus produzieren bestärkt, insoweit auch mit diesem Begriff die prozesshafte Herstellung von etwas (in diesem Fall die Geschlechterunterscheidung) umschrieben wird. Dieser Herstellungsprozess der 
Geschlechterunterscheidung wird dadurch charakterisiert, dass er sich fortlaufend vollzieht (,ständig wieder neu“) und die Unterscheidung nach gender somit - im Gegensatz zur Unterscheidung nach Geschlecht - keine unabänderliche Gegebenheit darstellt. Schließlich verweist die Aussage, dass es sich bei gender um ein „soziales Gebilde“ handelt, darauf, dass diese Art der Unterscheidung durch die Individuen selbst produziert wird, da Sozialität grundlegend auf das aufeinander bezogene Handeln von Individuen verweist. Gender wird also als ein Produkt eines sozialen Herstellungsprozesses definiert, der die Unterscheidung von Individuen in Frauen und Männer zur Folge hat.

Im Kontext der Ausführungen zum Terminus gender wird im Material auch erklärt, dass man , in diesem Zusammenhang von der sozialen Geschlechterrolle“ spricht (ABK, J51). Der Begriff der Rolle findet sich zum einen im Kontext des Schauspiels und meint diesbezüglich die darstellende Verkörperung einer Figur. Zum anderen wird er innerhalb soziologischer Debatten - mitunter auch im Sinn einer metaphorischen Beschreibung der sozialen Welt als Theater - als ein Set von gesellschaftlichen Erwartungen zu (in diesem Kontext: auf Geschlecht bezogenen) Verhaltensweisen verhandelt, die an Personen herangetragen werden und die es von Personen einzunehmen und adäquat zu spielen gilt (Goffman 2007; Meuser 2010, S. 50-63). Mit dem Zusatz „sozial““ im Zusammenhang mit den möglichen Deutungen des Terminus Rolle wird die Lesart von gender als ein Produkt eines sozialen Konstruktionsprozesses erneut bestärkt, denn gender wie auch die soziale Geschlechterrolle implizieren individuell umzusetzende Handlungsweisen, die die Hervorbringung des Geschlechterunterschieds zur Folge haben.

Der Ursprung von Geschlechterrollen wird im Datenmaterial in der „Urzeit“ verortet (ABK, J53). Die ,urzeitliche Rolle[n]“ sahen demnach den Mann als „Jäger“ und die Frau als „Sammlerin und Brutversorgerin“ vor (ABK, J53). Aus dieser Rollenverteilung sei historisch die Gesellschaftsform des Patriarchats erwachsen. Diese spezifische Organisationsform einer Gesellschaft und die darin inbegriffenen „traditionellen Rollenzuschreibungen sozialer Geschlechterrollen“ (ABK, J53) führten nach den Verfassenden zu einer gesellschaftlichen Sphärenteilung, in der sich die private Sphäre als weiblicher und die öffentliche Sphäre als männlicher Raum etablierten. Aus dieser Sphärentrennung habe sich auch eine geschlechtstypische Aufgabenteilung entwickelt, in der der Mann als das „Oberhaupt und Ernährer der Frau und Familie“ fungiert und in der die Frau die Zuständigkeit ,für die sozialen Bindungen innerhalb der Partnerschaft und der Familie“ innehabe (ABK, J53). Diesem Arrangement wird von den Verfassenden eine noch immerwährende Aktualität zuerkannt, insoweit in ,den 
meisten modernen westlichen Gesellschaften [...] diese Rollenzuschreibungen immer noch zu[treffen]“ (ABK, J54).

Vor dem Hintergrund der bisherigen Rekonstruktion des diskursiven Geschlechterwissens, das im Material vermittelt wird, soll nun zunächst ein erstes Resümee gezogen werden. Das diskursive Geschlechterwissen ist vor allem geprägt durch die Annahme, dass die soziale Wirklichkeit zweigeschlechtlich strukturiert ist, denn den materialimmanenten Verständnissen von Geschlecht und gender liegt die Unterteilung in Frau/Mann sowie in weiblich/männlich zugrunde. Dieser Umstand wird im Material jedoch nicht reflektiert, d. h. die Geschlechterdifferenz wird nicht als eine sich fortlaufend vollziehende soziale Praxis dargestellt, sondern als essenzielle Eigenschaft von Individuen gefasst (vor allem durch den Verweis auf den biologischen Geschlechterunterschied). Anstelle einer Vermittlung des Konzepts des doing gender wird doing gender vielmehr betrieben, denn die Geschlechterunterscheidung wird im Material immer wieder unreflektiert produziert.

In Bezug auf das Konzept des undoing gender lässt sich für die Rekonstruktionen aus dem Material festhalten, dass a) keine Geschlechtergleichheit artikuliert, sondern die Geschlechterdifferenz vielmehr betont wird, dass b) keine Änderungen bestehender Geschlechternormen angestrebt werden, sondern stattdessen deren Aktualität hervorgehoben wird, und dass sich c) keine Unterlassung von oder Widerständigkeit gegen die Praxis der Geschlechterunterscheidung ausmachen lässt, sondern dass diese über die Thematisierung der biologischen Fortpflanzung vielmehr sinnhaft aufgeladen wird. Daher lassen die bisherigen Rekonstruktionen zum materialimmanenten diskursiven Geschlechterwissen keine Rückschlüsse auf ein undoing gender zu.

Im Anschluss an die dargestellten Rekonstruktionen des diskursiven Geschlechterwissens, soll dieses Wissen nun mit gegenwärtigen Annahmen der Geschlechtersoziologie konterkariert werden. Denn auch im Material selbst wird darauf verwiesen, dass „,neuere Ergebnisse der Geschlechterforschung berücksichtigt werden [müssen]“ (ABK, J59). Mit der Bezeichnung gender wird semantisch auf die geschlechtersoziologische sex-gender-Unterscheidung verwiesen. Diese Unterscheidung hat ihren Ursprung in sexualwissenschaftlichen Studien der 1950er- und 1960er-Jahre zu transsexuellen Personen (als bekanntestes Beispiel sei hier auf Garfinkels Studie (1967) zur transsexuellen Agnes verwiesen). Mit der Unterscheidung von sex und gender wurde beabsichtigt, semantisch zu fassen, dass die anatomische Geschlechtszugehörigkeit nicht notwendigerweise mit der empfundenen Geschlechtsidentität übereinstimmen müssen: 
In der sex/gender-Unterscheidung bezog sich die Kategorie ,sex“ nun allein auf das ,biologische Geschlecht‘ (Anatomie, Physiologie, Morphologie, Hormone, Chromosomen). Die Kategorie ,gender' dagegen zielte auf das ,soziale Geschlecht" im Sinne der sozialen und kulturellen Prägung von Verhaltenserwartungen, Eigenschaftszuschreibungen, Tätigkeiten und darauf bezogenen sozialen Positionierungen.

(Gildemeister und Hericks 2012, S. 189)

Im Datenmaterial wird Geschlecht sehr ähnlich zum Begriff sex als eine biologisch bedingte Unterscheidung in weibliche und männliche Individuen gefasst, die vor allem an körperlichen Merkmalen orientiert ist. Auch der Begriff gender wird im Material in ähnlicher Weise zum gleichnamigen, geschlechtersoziologischen Begriff definiert. Denn auch im Material wird beim Terminus gender dessen sozialkonstruktivistischer Charakter hervorgehoben. Ein wesentlicher Unterschied zwischen dem materialinternen und dem geschlechtersoziologischen gender-Begriff besteht jedoch darin, dass gender im Datenmaterial als eine Erweiterung zum rein biologisch determinierten Verständnis von Geschlecht dargestellt wird. Darüber hinaus wird gender im Ausbildungsmaterial vor allem mit typischen Eigenschaften und Verhaltensweisen von Frauen und Männer beschrieben. Allein jedoch zu wissen, ,dass Lippenstift, Make-up und Barbie-Wahn ebenso wenig wie das Kopftuchgebot zur ,Natur der Frau“ gehören“, fasst aus geschlechtersoziologischer Perspektive noch nicht die Annahme einer sozialen Konstruiertheit von Geschlecht, sondern lediglich ein Wissen über ,kulturelle Variationen in der Ausgestaltung von Geschlechterbildern" (Gildemeister und Hericks 2012, S. 196).

Die Absicht konstruktivistischer Ansätze der Geschlechtersoziologie ist es dagegen, das Phänomen der Zweigeschlechtlichkeit als binäres Ordnungssystem der Gesellschaft in den Blick zu nehmen (Lenz und Adler 2010). Diese Perspektive der Geschlechtersoziologie entwickelte sich in einer kritischen Auseinandersetzung mit der sex-gender-Unterscheidung. Die Kritik bezog sich darauf, dass trotz der Erweiterung um eine soziale Dimension von Geschlecht die Bipolarität der Kategorie Geschlecht (weiblich/männlich) bestehen bliebe (Butler 1991; Gildemeister und Wetterer 1992). Innerhalb geschlechtersoziologischer Debatten wird mit der Konstruiertheit von Geschlecht betont, dass nicht nur gender, sondern auch das biologische Geschlecht ein soziales Konstrukt sei, insoweit aus Genitalien allein ,keine Geschlechterordnung entsteht, sondern erst aus einer Geschlechterordnung heraus Genitalien mit Bedeutung aufgeladen und zu ,Geschlechtszeichen“ werden können“ (Gildemeister und Hericks 2012, S. 197, Hervorhebung im Original). Somit wird aus einer geschlechtersoziologischen Perspektive mit der Konstruiertheit von Geschlecht auf die wechselseitige Konstituierung von sex und gender hingewiesen. 
Zwar findet sich auch im Datenmaterial, zumindest terminologisch, die Idee der Konstruiertheit von Geschlecht, etwa wenn festgehalten wird: „Das Gender ist ,konstruiert“" (ABK, J52). Jedoch wird im analysierten Ausbildungsmaterial dem ausschließlich biologisch bestimmten Geschlecht kein vergleichbarer Konstruktionscharakter zugeschrieben, wie er innerhalb der Geschlechtersoziologie vertreten wird, sondern Geschlecht wird materialintern als naturalistische, essenzielle, unabänderliche biologische Gegebenheit definiert. In der datenmaterialinternen Argumentation, dass die Geschlecht-gender-Unterscheidung letztlich auf urzeitliche Geschlechterrollen (Jäger/Sammlerin) zurückgehe, wird viel eher eine kausale Verbindung (statt einer wechselseitigen Konstituierung) von biologischem und sozialem Geschlecht provoziert.

Die Annahme über die urzeitliche Geschlechtersphärentrennung von dem Mann als Jäger und der Frau als Sammlerin wird überdies innerhalb gegenwärtiger Forschungsarbeiten mindestens infrage gestellt (Kästner 2012). Darüber hinaus sind naturwissenschaftliche Wissensbestände mitunter durch vergeschlechtlichte Deutungen geprägt, wie etwa Sigrid Schmitz (2006) aufzeigt. Nach Schmitz werden historische Artefakte retrospektiv interpretiert, allerdings erfolge die Deutung auf der Grundlage des Wissens der gegenwärtigen Gesellschaft. So könnten etwa drei unterschiedlich große, nebeneinander angeordnete Fußabdrücke auf unterschiedlichste Art und Weise interpretiert werden. Mit einer Deutungsfolie gegenwärtigen Wissens (zu Geschlecht) sehe man darin allerdings umgehend eine Familie aus Mutter, Vater und Kind (Schmitz 2006). Dieser Überlegung folgend können auch die Jäger-Sammlerin-Unterscheidung und die damit implizierte geschlechtliche Sphärentrennung als ein mögliches Produkt vergeschlechtlichter Deutung gesehen werden.

Hinsichtlich des materialimmanenten diskursiven Geschlechterwissens lässt sich vor dem Hintergrund der Einbeziehung gegenwärtiger geschlechtersoziologischer Debatten schlussfolgern, dass im Material zwar ein Wissen um die Konstruiertheit von gender postuliert wird, dass jedoch über die im Material vorgenommene biologistische Fundierung jede Form von Geschlechtlichkeit (ob biologisch oder sozial) letztendlich als zwei-geschlechtlich dargestellt wird. Entsprechend findet im Material doing gender vielmehr statt, als dass es als Konzept reflektierend vermittelt wird. Ansätze eines undoing gender werden aufgrund des geschlechtlichen Essenzialismus schließlich verunmöglicht, da jede Idee einer (De-)Konstruktion von Geschlecht durch die im Material gegebene biologistische Fundierung verunmöglicht wird.

Im Anschluss an das rekonstruierte diskursive Geschlechterwissen, das dem Ausbildungsmaterial Fachfrau/Fachmann Betreuung zugrunde liegt und der Kontrastierung dieses Geschlechterwissens mit gegenwärtigen Erkenntnissen der 
Geschlechtersoziologie, soll sich nun dem Aspekt der Vermittlung dieses Wissens innerhalb des Datenmaterials zugewendet werden. Für dieses Vorhaben wird zunächst dargestellt, welche richtungsweisenden Aspekte im Material unter einer ,genderspezifischen Betreuung“(ABK, J58) gefasst werden und welche grundlegenden Handlungsanweisungen diesbezüglich gegeben werden. Daran anschließend wird die Analyse zu verschiedenen, imaginierten Praxisbeispielen aus dem Alltag von pädagogischen Fachkräften vorgestellt, um das im Material vermittelte, handlungspraktische Geschlechterwissen zu rekonstruieren.

\subsubsection{Theorie $\neq$ Praxis - das handlungspraktische Geschlechterwissen zwischen Reflexion und Reproduktion der Geschlechterdifferenzierung}

Für die Rekonstruktion des handlungspraktischen Geschlechterwissens gibt bereits der Abschnitt „Gender - Männer weinen nicht“ erste Hinweise, da in diesem auf Vorstellungen zu einer genderspezifischen Betreuung eingegangen wird. Ein erster Schwerpunkt in der Darstellung einer genderspezifischen Betreuung befasst sich mit der Kita als räumliches und ideelles Setting. So wird darauf verwiesen, dass unreflektierte Geschlechtsstereotype den Kita-Alltag prägen, was sich von der Raumgestaltung bis zur Bewertung (und Sanktionierung) der Verhaltensweisen der Kinder ziehen könne (ABK, J58). Als Möglichkeiten einer bewusst(er)en Gestaltung des Kita-Alltag wird die Methode der ,Geschlechterbrille“ angeführt (ABK, J60), über deren metaphorisches Aufsetzen man sehen könne, ob/wie Befindlichkeiten von Kindern (bei der Raumnutzung oder bei Verhaltensweisen) im Kontext ihrer Geschlechtszugehörigkeit stehen.

Ein zweiter Schwerpunkt der Prämissen für eine genderspezifische Betreuung liegt auf den pädagogischen Fachkräften bzw. auf der Arbeit im pädagogischen Team. Eine genderspezifische Betreuung könne darüber erreicht werden, dass man ,bei sich selbst“ anfange und ,eine Grundhaltung aufbauen“ solle, worunter eine Selbstreflexion in Bezug auf die eigene Geschlechter- und Berufsrolle gefasst wird (ABK, J59). Darüber hinaus wird vorgeschlagen, dass sich Fachfrauen/Fachmänner ,,von Zeit zu Zeit ,genderuntypisch“" verhalten könnten, wobei nicht ausgeführt wird, was damit konkret gemeint ist (ABK, J59). Schließlich wird betont, dass innerhalb des Teams eine gemeinsame ,ethische Haltung“ gegenüber der ,genderspezifischen Betreuung“ erarbeitet werden solle (ABK, J59).

Ein dritter Schwerpunkt liegt schließlich auf dem Umgang mit den zu betreuenden Kindern. Es wird betont, dass die Selbstbestimmung und die individuellen 
Neigungen der Kinder geachtet werden sollen und dass die Unterschiedlichkeit von Jungen und Mädchen stets zu berücksichtigen sei (ABK, J59). Pädagogische Fachkräfte sollen entsprechend zu diesen Vorgaben aufmerksam begleiten, wie Mädchen und Jungen sich mit Weiblichkeit und Männlichkeit auseinandersetzen und sie sollen die Kinder auch nicht daran hindern, sich auch mal genderuntypisch zu verhalten bzw. die Kinder dazu sogar motivieren.

Aus diesen im Material gesetzten Prämissen zu einer genderspezifischen Betreuung lassen sich bereits einige Hinweise auf das zu rekonstruierende, handlungspraktische Wissen ausmachen. So wird das Thema gender ganz grundlegend als für die berufliche Handlungspraxis bedeutsam angesehen, was daran deutlich wird, dass mehrfach dazu aufgefordert wird, als Team und für sich eine Haltung gegenüber dem Thema zu entwickeln. Darüber hinaus lässt sich zum einen ein Hinweis auf eine erhöhte Sensibilität gegenüber einem alltäglichen doing gender ausmachen (Methode der Geschlechterbrille), zum anderen lässt sich sogar ein Hinweis auf undoing gender im Sinn einer Änderung gängiger Geschlechternormen finden (Motivation zu genderuntypischem Verhalten). Inwieweit diese Prämissen (wie auch das diskursive Geschlechterwissen) in der (fiktionalen) Alltagspraxis Anwendung finden, wird erst die Analyse der Praxissituationen und der Rekonstruktion des handlungspraktischen Geschlechterwissens zeigen.

Die Analyse der Praxissituationen im Datenmaterial untergliedert sich nach den jeweiligen Personengruppen, die in den Praxissituationen dargestellt wurden. Es wurden die Darstellungen von Mädchen und Jungen, Fachfrauen und Fachmännern Betreuung sowie von Müttern und Vätern rekonstruiert. Im Folgenden wird die Analyse zweier Praxissituationen vorgestellt, in denen der Fokus auf der Interaktion zwischen pädagogischen Fachpersonen und Kindern lag:

Praxissituation - Die 3-jährige Lea ist umgefallen und hat sich das Knie aufgeschürft. Sie weint bitterlich und geht zur Betreuerin. Diese nimmt sie auf den Arm und tröstet sie mit einem Lied, zugleich gibt sie Lea, Tränchen', ein Fabelwesen mit einer großen Nase, die aussieht wie eine Träne, in die Hand. Diese schließt es sofort in ihre Arme und lässt sich bald beruhigen. Die Betreuerin und das ,Tränchen“ spenden beide Trost. (KIN A 31)

Bei Lea handelt es sich dem Namen nach um ein Mädchen, das sich, resultierend aus einem Sturz, eine Verletzung zugezogen hat (,Knie aufgeschürft“). Das Mädchen weint, wahrscheinlich wegen des physischen Schmerzes, den sie in Folge des Sturzes erfährt. Die Ergänzung um das Adjektiv bitterlich verweist auf ein intensives Empfinden von Schmerz. Das Mädchen löst ihre Problemsituation darüber, dass sie den Kontakt zur weiblichen Betreuungsperson sucht (,geht zur Betreuerin“). Die Reaktion der Betreuerin besteht aus mehreren Umgangsweisen: 
sie nimmt zunächst körperlichen Kontakt auf (,,auf den Arm“), sie kontaktiert das Kind aber auch verbal, indem sie sie durch das Singen eines Lieds tröstet. Das körperliche und verbale Trösten wird darüber hinaus mit der Gabe eines Kuscheltiers ergänzt. Insgesamt zeigt sich in der Reaktion der Fachfrau Betreuung eine intensive Fürsorglichkeit wie auch eine emotionale Anteilnahme an der Situation des Kindes.

Die Fachfrau Betreuung geht nicht auf die eigentliche Problemsituation ein, indem sie etwa die Schwere der Verletzung prüft oder erfragt, wie es zu der Verletzung kam. Sie geht jedoch auf die durch die Problemsituation verursachte Gefühlslage des Mädchens ein und vermittelt damit auch, dass sie die Empfindungen des Mädchens ernst nimmt. Dass diese Vorgehensweise von dem Mädchen angenommen wird, zeigt sich schließlich darin, dass sie sich beruhigen lässt. Somit wird die Problemsituation durch die Vorgehensweise der Fachfrau Betreuung für das Mädchen mit einem positiven Ausgang abgeschlossen. Innerhalb des Ausbildungsmaterials wurde eine weitere Darstellung einer Praxissituation gefunden, die der eben vorgestellten sehr ähnlich war - mit dem Unterschied, dass es diesmal nicht um ein Mädchen, sondern um einen Jungen ging:

Praxissituation - Ueli stürzt im Kinderhort mit dem Dreirad. Er ist dabei ein wenig erschrocken und weint. Sein Knie ist leicht lädiert. Da kommt Sabina Meier, die Fachfrau Betreuung, angerannt. Sie nimmt ihn auf den Arm und tröstet ihn: ,Komm Ueli, es ist nicht so schlimm. Sei tapfer, Knaben weinen nicht!‘(ABK J50)

Wie auch in der Praxissituation mit dem Mädchen ereignet sich in dieser beispielhaften Beschreibung ein Sturz, der eine Verletzung am Knie zur Folge hat. Die Reaktion von Ueli auf dieses Ereignis besteht, ebenso wie bei Lea, im Weinen. Anders als bei dem vorangegangenen Beispiel nimmt der Junge jedoch nicht selbst Kontakt mit einer Betreuungsperson auf, sondern diese kommt in Reaktion auf den Sturz ,angerannt“, was eine gewisse Eile oder Hektik und damit wiederum eine antizipierte Dramatik der Situation impliziert. Wie auch bei der Situation des gestürzten Mädchens ist in diesem Beispiel die Reaktion der Fachfrau Betreuung auf den Sturz geprägt durch eine körperliche Kontaktaufnahme (,nimmt ihn auf den Arm“). Dies vermittelt zunächst auch ein fürsorgliches, zugewandtes, tröstendes Handeln der Betreuerin - ähnlich dem Beispiel des gestürzten und aufgrund dessen weinenden Mädchens.

Es erfolgt, wie auch in der zuvor dargestellten Praxissituation, im Anschluss an die körperliche, auch eine verbale Kontaktaufnahme von der Fachfrau Betreuung zu dem Kind. Dem Jungen wird zunächst gesagt, dass es ,nicht so schlimm“ sei. Mit dieser Aussage wird deutlich gemacht, dass die Fachfrau Betreuung die 
Gefühlslage des Jungen nicht teilt - aus ihrer Perspektive ist die Situation und deren Folge eben nicht so schlimm (wie vom Jungen empfunden und über das Weinen ausgedrückt). Es folgt die in einer auffordernden Art und Weise formulierte Aussage: „Sei tapfer“. Der Begriff tapfer steht zum einen für eine Furchtlosigkeit, zum anderen - und vor allem im Kontext von Kindern - wird der Begriff aber auch verwendet, um eine Beherrschtheit zu beschreiben, die sich darin ausdrückt, dass Emotionen nicht nach außen getragen, sondern nach innen verdeckt werden. Entsprechend dieser Lesart fordert die Fachfrau Betreuung den Jungen also dazu auf, seine Empfindungen zu unterdrücken bzw. ihrer Wahrnehmung der Situation anzupassen.

Im Folgenden wird eine plausibilisierende Weiterführung der bisherigen Aussage vorgenommen, indem die Fachfrau Kinderbetreuung sagt „Knaben weinen nicht“. Dass sie die Pluralform Knaben wählt, zeigt zum einen an, dass sie zwar weiterhin Ueli adressiert, sie nun aber zum anderen auf eine abstraktere Ebene wechselt. Ueli bildet zwar noch den Ausgangspunkt, aber es geht ihr nicht mehr um Ueli in seiner individuell erfahrenen Situation. Denn vielmehr wird er mit dieser Aussage einer größeren sozialen Gruppierung zugeordnet (den Knaben). Für Zugehörige dieser sozialen Gruppe wird generalisierend ein spezifisches Charakteristikum konstatiert: Knaben würden nicht weinen. Weinen stellt einen Ausdruck von Emotionalität dar (ob vor Freude, körperlichem Schmerz oder Traurigkeit), der sich in der Regel unbeabsichtigt vollzieht. Nicht zu weinen vermittelt also im Umkehrschluss, dass Emotionen von Zugehörigen dieser Gruppe bewusst nicht gezeigt bzw. unterdrückt werden. Es handelt sich bei der Aussage also weniger um die Beschreibung eines Sachverhalts, sondern viel eher um die Formulierung einer sozialen Norm: Knaben sollten nicht weinen. Diese antizipierte Norm hat schließlich einen dezidiert geschlechtsspezifischen Charakter, insoweit sie exklusiv auf eine soziale Gruppe angewendet wird, die durch die Geschlechtszugehörigkeit semantisch definiert wird (Knaben).

Die Fachfrau Betreuung wendet sich mit dieser sozialen Norm (Knaben weinen nicht) an den Jungen Ueli, der gerade genau das tut, was nicht der Norm entspricht (als Knabe weinen). Damit impliziert sie, dass sich Ueli mit seinem situativen Verhalten nicht sozialkonform und daran anschließend nicht geschlechtskonform verhalte. Da dieser Normdarstellung eine imperative Formulierung vorausging (,Sei tapfer"), ist anzunehmen, dass es sich bei dieser nachgeschobenen Aussage auch um eine implizite Aufforderung handelt. Mit der Konstatierung dieser geschlechtsspezifischen Norm wird dem Jungen also implizit die Handlungsanweisung gegeben, seine Verhaltensweise sozial- und geschlechtskonformer zu gestalten - er soll nicht weinen, denn er ist ein Knabe. 
Im Gegensatz zum Beispiel des ebenfalls gestürzten und weinenden Mädchens, werden die Empfindungen und die Wahrnehmung des Jungen in dieser illustrierten Situation von der Fachfrau Betreuung nicht ins Zentrum ihres Vorgehens gerückt. Während die pädagogische Fachkraft im Beispiel mit dem gestürzten Mädchen explizit auf deren Gefühlslage eingeht, wird dem Jungen vergleichsweise weniger empathische Fürsorge durch die Betreuungsperson zuteil. Darüber hinaus wird sein Verhalten über die Formulierung einer indirekten Handlungsanweisung durch die Fachkraft als normative Abweichung gerahmt.

Im Ausbildungsmaterial wird also bei fast identischen Problemsituationen (Stürzen) und sehr ähnlichen Reaktionen der Kinder auf diese Situation (Weinen aufgrund eines Sturzes) ein wesentlicher Unterschied im fachpädagogischen Umgang dargestellt (Trösten vs. Normierung). Der Unterschied im Umgang mit den Kindern scheint in den beschriebenen Situationen auf spezifischen Vorstellungen adäquater Verhaltensweisen von Mädchen und Jungen und damit wiederum auf antizipierten Geschlechtervorstellungen und -attribuierungen $\mathrm{zu}$ basieren. In den beiden Praxissituationen des Datenmaterials wird folglich ein doing gender betrieben (statt reflektiert), insoweit die Geschlechterunterscheidung zum Ausgangspunkt im sozialen Miteinander mit den Kindern und im Unterscheiden von Kindern wird.

Die Darstellung von auf Geschlecht basierenden, unterschiedlichen Wahrnehmungen, Bewertungen und Umgangsweisen durchziehen die gesamten analysierten Praxissituationen im Ausbildungsmaterial von Fachfrau/Fachmann Betreuung. Dies betrifft unter anderem die Darstellungen selbstgewählter oder angeregter Motiv-/Spielzeugwahlen von Kindern, die illustrierten Verhaltensweisen von Kindern miteinander und die Umgangsweisen der Fachkräfte mit Kindern in Konfliktsituationen. Die Kinder wählen in den im Ausbildungsmaterial gegebenen Beispielen vermeintlich geschlechtskonforme Beschäftigungen und Themen (beispielsweise Kätzchen vs. Panther), Jungen werden überwiegend als potenzielle Verursacher von Konflikten beschrieben und die Freundschaft zwischen Jungen und Mädchen wird als romantisierte, heteronormative Zweierbeziehung dargestellt. Die Fachkräfte Betreuung werden im Material überwiegend als Frauen thematisiert, die geschlechtsdifferenzierende Verhaltensweisen unterstützen bzw. diese sogar fördern.

Darüber hinaus lassen sich weder genderuntypisches Verhalten oder Anregungen dazu ausmachen, noch werden eine (Selbst-)Reflexion über Geschlechtsstereotype thematisiert oder Anregungen zu einer bewusst(eren) Gestaltung des Kita-Alltages gegeben. Vor dem Hintergrund dieser Erkenntnisse lässt sich schlussfolgern, dass weder die formulierten Prämissen für eine genderspezifische Betreuung (mit Optionen zur Reflexion des doing gender und Ansätzen zum 
undoing gender), noch das diskursive Geschlechterwissen zur Konstruiertheit von gender Eingang in die im Material gegebenen Praxissituationen finden. Das handlungspraktische Geschlechterwissen, das im Material vermittelt wird, ist also vor allem durch ein beständiges doing gender geprägt, denn die Geschlechterunterscheidung (zwischen weiblichen und männlichen Fachkräften, zwischen Mädchen und Jungen sowie zwischen Müttern und Vätern) wird immer wieder aufs Neue reproduziert.

Was sich dagegen deutlich während der Analysen abzeichnete, ist eine Respektierung der Selbstbestimmung der Kinder durch die pädagogischen Fachkräfte. Dies entspricht wiederum anderen Prämissen der Betreuungsarbeit, die darin festgemacht werden, dass „Mädchen und Jungen [...] in ihren individuellen Neigungen und Interessen zu unterstützen“ und dass „Kinder und Jugendliche [als] aktive Gestalter ihrer Entwicklung [...] ernst zu nehmen“ seien (ABK, J59). Das rekonstruierte doing gender bzw. die Beförderung dessen durch pädagogische Fachkräfte verläuft somit entlang einer anderen, zentral formulierten pädagogischen Aufgabe: dem Fördern und Respektieren der Selbstbestimmung der zu betreuenden Kinder.

Auf die Problematik konkurrierender pädagogischer Aufgaben haben bereits Glenda MacNaughton (2000) und Naima Browne (2004) hingewiesen. Beide Autorinnen diskutieren kritisch, dass eine entwicklungspsychologisch begründete Fokussierung auf die Förderung der Individualität von Mädchen und Jungen systematisch eine Berücksichtigung der Effekte der gesellschaftlich-kulturellen Konstruktion von Geschlecht verhindert (Browne 2004; MacNaughton 2000). Dies bedeutet, dass das pädagogische Ziel der Förderung der Individualität von Kindern unvereinbar mit einer Reflexion über Prozesse des doing gender ist, da letztere mitunter eine Einflussnahme auf die Selbstentfaltung der Kinder nach sich zieht. Sinngemäß dazu kann auch für die Analysen des Materials geschlussfolgert werden, dass sich die materialimmanenten Zielstellungen (Selbstbestimmung der Kinder - Reflexion der Konstruiertheit von gender) gegenseitig verunmöglichen. Denn durch die Betonung des selbstbestimmten Handelns und Entfaltens von Kindern werden Reflexionen zu und gegebenenfalls ein Wirken gegen Prozesse des doing gender schon in der Anlage des Ausbildungsmaterial für Fachfrau/Fachmann Kinderbetreuung verhindert. 


\subsection{Rhetorisch modernisiert? Zur Verortung des rekonstruierten Geschlechterwissens in geschlechtersoziologischen Kontexten}

In einem abschließenden Schritt werden nun die zentralen Ergebnisse zum rekonstruierten diskursiven sowie zum handlungspraktischen Geschlechterwissen aus den exemplarisch dargestellten Analysen zusammengeführt und theoretisch rückgebunden. Strukturiert wird dieser finale Schritt über die schrittweise Beantwortung der eingangs formulierten Forschungsfragen, die für die Analyse des Ausbildungsmaterials Fachfrau/Fachmann Kinderbetreuung anleitend waren.

\subsubsection{Welches Geschlechterwissen wird im Ausbildungsmaterial zu Fachfrau/Fachmann Kinderbetreuung vermittelt?}

Im Ausbildungsmaterial Fachfrau/Fachmann Betreuung ließen sich zwei Formen von Geschlechterwissen rekonstruieren: ein diskursives, didaktisch aufbereitetes Wissen über Geschlechterwissen sowie ein an der beruflichen Alltagspraxis orientiertes, handlungspraktisches Geschlechterwissen. Wie aufgezeigt wurde, weicht das rekonstruierte diskursive Geschlechterwissen von etablierten Annahmen der Geschlechtersoziologie ab und das obwohl innerhalb des Materials als Zielsetzung festgehalten wird, dass „neuere Ergebnisse der Geschlechterforschung berücksichtigt werden [müssen]“ (ABK, J59).

Mit diesem Anspruch wird noch einmal verdeutlich, dass das Ausbildungsmaterial als eine Form des Schul- bzw. Lehrbuchs gerahmt werden kann, denn ein Spezifikum dieser Datensorte besteht unter anderem in der Vermittlung aktueller, fachwissenschaftlicher Forschungserkenntnisse zu einem bestimmten Thema. Wie aufgezeigt wurde, nehmen die Autorinnen und Autoren des Ausbildungsmaterials zwar semantisch über die Begriffe Geschlecht und gender auf gegenwärtige theoretische Debatten der Geschlechtersoziologie Bezug, jedoch wurde anhand der Analyse auch deutlich, dass das materialimmanente diskursive Geschlechterwissen vom etablierten Verständnis der Geschlechtersoziologie differiert, da gender lediglich als eine Erweiterung zum biologistisch fundierten Geschlechtsbegriff plausibilisiert wird.

Innerhalb der Geschlechtersoziologie wurde mit der Einführung der sexgender-Unterscheidung beabsichtigt, die Geschlechtsidentität nicht länger als Resultat der biologischen Geschlechtszugehörigkeit und die Ungleichheitsverhältnisse zwischen den Geschlechtern nicht länger als Effekt der anatomischen 
Unterschiede zu fassen. Mit dem sex-gender-Modell konnte die soziale Ordnung als ein Ergebnis von kulturellen und sozialen Prozessen erklärt und kritisiert werden. Was sich mit dieser Unterscheidung auftat, war eine Differenzierung zwischen Natur (biologisches Geschlecht) und Kultur (soziales Geschlecht), „wobei die Biologie (Natur) als unveränderlich und statisch galt, die kulturellen Ausprägungen dagegen als kulturell und historisch spezifisch und daher wandelbar“ (Gildemeister und Hericks 2012, S. 190).

Doch innerhalb der Geschlechtersoziologie entwickelten sich in den letzten Jahrzehnten vielerlei Zweifel und Kritik in Bezug auf das sex-gender-Modell, da etwa die Unterschiede innerhalb eines Geschlechts keine Berücksichtigung fanden, die Beziehung zwischen Natur und Kultur unreflektiert blieb und nicht zuletzt die Geschlechterdifferenz zwar um die soziale Dimension erweitert, aber das biologische Geschlecht weiterhin als naturgegeben verstanden wurde. Aus der Annahme eines naturgegeben biologischen Geschlechts ergibt sich, dass zumindest ein Teil der Geschlechterunterscheidung weiterhin der Natur zuzuordnen sei, was Gildemeister und Wetterer als „verlagerten Biologismus“ bzw. als „latenten Biologismus der Gesamtkonstruktion“ (Gildemeister und Wetterer 1992, S. 206 f.) bezeichnen. Der Effekt eines sex-gender-Modells, in dem das biologische Geschlecht als statisch und essenziell gefasst wird, besteht darin, dass ein solches Modell stets doch auf ein biologistisches Argumentationsmuster zurückfallen kann, d. h. das ein solches Modell letztlich immer vermeintlich natürliche Unterschiede zwischen Mann und Frau (und Mädchen und Jungen) hervorbringt.

Genau dieses sex-gender-Modell, in dem das biologische Geschlecht essenzialistisch verstanden wird, liegt auch dem rekonstruierten diskursiven Geschlechterwissen zugrunde. Im Material wird zwar der Konstruktionscharakter von gender hervorgehoben, aber durch die Annahme, dass das biologische Geschlecht unabänderlich ist und durch die Historisierung dieser Annahme (Jäger-SammlerinUnterscheidung) wird letztendlich ein latenter Biologismus im Sinn Gildemeister und Wetterers fortgeschrieben. Das biologische Geschlecht und damit einhergehende Geschlechterattribuierungen werden so als vermeintliche Tatsachen plausibilisiert und damit jeglicher Hinterfragung entzogen. Durch ein solches Verständnis von Geschlecht und gender entsteht somit der Eindruck, dass das Geschlechterverhältnis schon immer so gewesen sei und immer so sein werde, denn Frauen (und Mädchen) wie Männer (und Jungen) sind diesem Verständnis nach von Natur aus so. Dieser Effekt spiegelt sich schließlich auch in den Analysen der Praxissituationen wieder, denn wie aufgezeigt wurde, ist das handlungspraktische Geschlechterwissen geprägt durch ein beständiges doing gender, in dem die Praxis der Geschlechterunterscheidung unreflektiert bleibt. 


\subsubsection{Welche geschlechtsspezifischen Aspekte werden im Lehrmaterial thematisiert und auf welche Weise werden diese verhandelt?}

Das zu rekonstruierende Geschlechterwissen wurde für die Analyse in Anschluss an Wetterer in ein diskursives und ein handlungspraktisches Wissen dimensionalisiert. Wie der Vergleich der beiden rekonstruierten Wissensarten zu Geschlecht im Datenmaterial zutage förderte, besteht ein Gap zwischen diesen, denn das materialimmanente, diskursive Geschlechterwissen (wie auch die im Material formulierten Prämissen einer genderspezifischen Betreuung) werden in keiner Weise in den (imaginierten) Praxissituationen reflektiert oder umgesetzt.

Einen ersten Erklärungsansatz für diesen Gap bietet die Überlegung Wetterers, dass sich im Zuge der rhetorischen Modernisierung eine ,Semantik der Gleichheit" (Gleichberechtigung zwischen Frauen und Männern) etabliert habe, die jedoch einer beharrlichen „Praxis der Differenzierung“ (Frauen und Männer sind unterschiedlich) gegenüberstehe (Wetterer 2008b, S. 46). Wetterer schlussfolgert aus dieser Gegenüberstellung von Diskurs und Praxis, dass die Modernisierung des Geschlechterverhältnisses rein rhetorischer Natur sei, denn in der alltagsweltlichen Praxis (Kinderbetreuung, Haushaltstätigkeiten, Berufswahl etc.) schreiben sich weiterhin tradierte geschlechtsspezifische Annahmen und Handlungsweisen fort, auch wenn sich die Idee einer Gleichheit von Frauen und Männern etabliert hat (Kassner 2008; Koppetsch und Burkhart 1999; Nentwich 2004).

Auch für die Analysen zum Geschlechterwissen im Ausbildungsmaterial für Fachfrau/Fachmann Kinderbetreuung lässt sich eine rhetorische Modernisierung im Sinn einer Diskrepanz zwischen Diskurs und Praxis konstatieren. Denn im materialimmanenten, diskursiven Geschlechterwissen wird zwar die Annahme der Konstruiertheit von gender propagiert, doch wird diese Annahme in der (fiktionalen) Alltagspraxis weder reflektierend berücksichtigt noch umgesetzt, sondern es verbleibt bei einer Fortschreibung der Geschlechterdifferenzierung. Die so entstehende Diskrepanz zwischen diskursivem Geschlechterwissen und handlungspraktischem Geschlechterwissen lässt sich sinngemäß als eine Form rhetorischer Modernisierung deuten: Es besteht die Annahme, dass gender konstruiert sei (Diskurs) und doch dominieren Geschlechterdifferenzierungen (weiterhin) die soziale Ordnung in den Darstellungen des Datenmaterials (Praxis).

Doch auch innerhalb des diskursiven Geschlechterwissens dominiert eine Diskrepanz, denn auch wenn die Annahme vertreten wird, dass gender konstruiert sei, so wird doch auch argumentiert und historisch plausibilisiert, dass das biologische Geschlecht weiterhin essenziell sei. Einen Erklärungsansatz für diese Diskrepanz bieten die Überlegungen Nentwichs (2004,2014), die an Wetterers Ausführungen 
anknüpft, diese empirisch basiert weiterführt und die These einer „diskursive[n] ,Gleichzeitigkeit" von Differenz und Gleichheit" konstatiert (Nentwich 2014, S. 52).

Nentwich argumentiert, dass die analytische Trennung zwischen diskursiver Rhetorik einerseits und handlungsbasierter Praxis anderseits zu kurz greife: Zum einen verkenne eine solch strenge Unterscheidung die wirklichkeitsgenerierende Wirkung von Sprache, zum anderen könne eine solche Trennung den Blick auf empirische Befunde frühzeitig verengen (Nentwich 2014, S. 51 f.). Wie Nentwich empirisch rekonstruktiv anhand von Interviews mit Kitaleitungen, die ebenfalls im Rahmen des Forschungsprojekts „Puppenstuben, Bauecken und Waldtage: (Un)doing gender in Kinderkrippen" durchgeführt wurden, aufzeigt, ergibt sich die von Wetterer konstatierte Diskrepanz von Gleichheit und Differenz bereits auf diskursiver Ebene (und nicht erst im Vergleich von Diskurs- und Praxisebene):

Während die Idee der Gleichheit [...] der Geschlechter stark im diskursiven Wissen der Krippenleitungen verankert ist, besteht daneben ein ebenso klar verankertes Wissen über Differenz, das zur Erklärung der bestehenden Stereotypen wie auch der wahrgenommenen Erfahrungen mit Buben und Mädchen im Krippenalltag herangezogen wird. (Nentwich 2014, S. 55)

Entsprechend dieser Erkenntnisse schlussfolgert Nentwich, dass nicht erst zwischen dem diskursiven und dem praktischen Wissen von Geschlecht eine Diskrepanz entstehe, sondern dass diese Paradoxie bereits auf der rein diskursiven Ebene vorhanden ist und sich demgemäß eine diskursive Gleichzeitigkeit von Gleichheit und Differenz ergebe (Nentwich 2004,2014).

An diese Überlegungen anschließend kann für das analysierte Ausbildungsmaterial für Fachfrau/Fachmann Betreuung geschlussfolgert werden, dass sich ebenfalls eine rhetorische Modernisierung abzeichnet, die sich nicht allein auf die Diskrepanz zwischen diskursiver Rhetorik und handlungsbasierter Praxis beschränken lässt. Vielmehr zeichnet sich bereits im rekonstruierten diskursiven Geschlechterwissen eine Gleichzeitigkeit ab. Doch betrifft diese Gleichzeitigkeit nicht die Aspekte Gleichheit und Differenz, sondern - in Anlehnung an Nentwichs These - die Aspekte der Konstruiertheit und Essenzialität von Geschlecht. Denn wie in der Analyse dargestellt wurde, wird zwar innerhalb des Materials betont, dass gender sozial konstruiert sei, es wird jedoch gleichzeitig betont, dass das biologische Geschlecht eine essenzielle Eigenschaft von Individuen sei, die Menschen in Frauen und Männer unterscheidet. Somit kommt es bereits im diskursiven Geschlechterwissen zu einer Gleichzeitigkeit von konstruktivistischen wie auch differenzproduzierenden Verständnissen von Geschlecht, die sich im 
handlungspraktischen Geschlechterwissen in einem doing gender im Sinn einer Reproduktion der (biologistisch begründeten) Geschlechterdifferenz fortschreibt.

\subsubsection{Wie werden Fachfrauen/Fachmänner Betreuung auf den Umgang mit Geschlechterthematiken in der beruflichen Praxis im Rahmen der Ausbildung vorbereitet?}

Die Beantwortung der dritten Forschungsfrage wird abschließend als Anlass für eine resümierende Zusammenführung der Analyseergebnisse genutzt. Die Konzeption und Vermittlung der materialimmanenten Formen von Geschlechterwissen ist durch gleich mehrere Widersprüche und Brüche geprägt. So wird Geschlecht zwar nicht ausschließlich biologistisch begründet, jedoch wird Geschlecht grundlegend und zuvorderst als biologistisch determiniert gefasst. Über diese biologistische Determination werden Ansätze für ein konstruktivistisches Verständnis von Geschlecht (das sich auch im Material findet) für die Praxis verunmöglicht, da im Zweifelsfall eben doch die biologische, vermeintlich natürliche Unterscheidung von Frauen und Männern zum Tragen kommt. Für die Praxis kann dies etwa bedeuten, dass Fachfrauen/Fachmänner zwar rhetorisch über den Konstruktionscharakter von Geschlechtsstereotypen zu Kindern oder im Team dank der Ausbildung Bescheid wissen, da jedoch ein biologistisches Verständnis von Geschlecht dominiert, besteht die Wahrscheinlichkeit, dass Geschlechtsstereotype letztlich doch als vermeintlich natürliche Tatsachen verstanden werden und sie entsprechend nicht reflektiert bzw. ihnen nicht entgegengewirkt wird.

Darüber hinaus finden sich zwar Anweisungen zu einer genderspezifischen Betreuung, die zur Transferierung des konstruktivistischen Geschlechterverständnisses in die Praxis dienen sollen. Es werden jedoch keinerlei konkrete Umsetzungsvorschläge gegeben, wodurch die angehenden Fachfrauen/Fachmänner zwar rhetorisch ausgebildet werden, in der konkreten Praxis jedoch auf sich selbst verwiesen bleiben. Unterstützt wird diese Tendenz nochmals dadurch, dass in der im Material konstruierten Alltagspraxis (den Praxissituationen) keine Reflexion oder Umsetzung der im Material gegebenen Anleitungen für eine genderspezifische Betreuung zu finden sind. Erschwert wird die Umsetzung einer im Material postulierten genderspezifischen Betreuung schließlich auch dahingehend, dass andere zentrale Ziele der pädagogischen Arbeit konträr dazu laufen. So kann etwa die Respektierung der Selbstbestimmung der Kinder mitunter dazu führen, dass von Kindern (re-)produzierten Geschlechterstereotypen nicht entgegengewirkt wird, da dies sonst die Entscheidungsfreiheit der Kinder einschränken würde. 
Somit lässt sich abschließend festhalten, dass das Ausbildungsmaterial angehende Fachfrauen/Fachmänner Betreuung rhetorisch auf den Umgang mit geschlechtsspezifischen Themen vorbereitet. Die pädagogischen Fachkräfte sind jedoch mit der (theoretischen wie praktischen) Plausibilisierung und Umsetzung der Brüche und Widersprüche gegenwärtiger Geschlechterverhältnisse und Wissensbestände zu Geschlecht auf sich allein gestellt, da diese im Ausbildungsmaterial selbst reproduziert werden.

\section{Literatur}

Barz, Monika (1982). Gleiche Chancen in Lesebüchern der Grundschule? In Ilse Brehmer (Hrsg.), Sexismus in der Schule. Der heimliche Lehrplan der Frauendiskriminierung (S. 103-114). Weinheim: Beltz.

Bauer, Alexandra (2007). Frauen und Mädchen in den Geschichtsbüchern der Hauptschulen - Ein aus der Mode gekommenes Thema? In Christin Grohn-Menard (Hrsg.), Bildung neu bilden. Balancen finden. 14. Bundeskongress Frauen und Schule (S. 423-442). Bielefeld: Kleine.

Bittner, Melanie (2015). Die Ordnung der Geschlechter in Schulbüchern. Heteronormativität und Genderkonstruktionen in Englisch- und Biologiebüchern. In Friederike Schmidt, Anne-Christin Schondelmayer, \& Ute B. Schröder (Hrsg.), Selbstbestimmung und Anerkennung sexueller und geschlechtlicher Vielfalt. Lebenswirklichkeiten, Forschungsergebnisse und Bildungsbausteine (S. 247-260). Wiesbaden: VS Verlag für Sozialwissenschaften.

Browne, Naima (2004). Gender Equity in the Early Years. Maidenhead: Open University Press.

Butler, Judith (1991). Das Unbehagen der Geschlechter. Frankfurt a. M.: Suhrkamp.

Butler, Judith (2004). Undoing Gender. London: Routledge.

Deutsch, Francine M. (2007). Undoing Gender. Gender \& Society, 21(1), 106-127.

Dölling, Irene (2005). Geschlechter-Wissen“ - Ein nützlicher Begriff für die ,verstehende" Analyse von Vergeschlechtlichungsprozessen? Zeitschrift für Frauenforschung \& Geschlechterstudien, 23(1+2), 44-62.

Elbracht, Marga, \& Mosler, Inge (1972). Femina non est homo? Zum Frauenbild in Grundund Hauptschullesebüchern. In Peter Braun (Hrsg.), Neue Lesebücher-Analyse und Kritik (S. 82-96). Düsseldorf: Bertelsmann.

Finsterwald, Monika, \& Ziegler, Albert (2007). Geschlechtsrollenerwartung vermittelt durch Schulbuchabbildungen der Grundschule. In Peter H. Ludwig \& Heidrun Ludwig (Hrsg.), Erwartungen in himmelblau und rosarot. Effekte, Determinanten und Konsequenzen von Geschlechterdifferenzen in der Schule (S. 117-143). Weinheim: Juventa.

Fried, Lilian, Dippelhofer-Stiem, Barbara, Honig, Michael-Sebastian, \& Liegle, Ludwig (2012). Pädagogik der frühen Kindheit. Weinheim: Beltz.

Garfinkel, Harold (1967). Passing and the Managed Achievement of Sex Status in an ,Intersexed Person'. In Harold Garfinkel (ed.), Studies in Ethnomethodology (pp. 116-185). Englewood Cliffs: Prentice-Hall. 
Gildemeister, Regine (2008). Soziale Konstruktion von Geschlecht: «Doing gender». In Sylvia M. Wilz. (Hrsg.), Geschlechterdifferenzen - Geschlechterdifferenzierungen. Ein Überblick über gesellschaftliche Entwicklungen und theoretische Positionen (S. 167-198). Wiesbaden: VS Verlag für Sozialwissenschaften.

Gildemeister, Regine, \& Hericks, Katja (2012). Geschlechtersoziologie. Theoretische Zugänge zu einer vertrackten Kategorie des Sozialen. München: Oldenbourg.

Gildemeister, Regine, \& Wetterer, Angelika (1992). Wie Geschlechter gemacht warden. Die soziale Konstruktion der Zweigeschlechtlichkeit und ihre Reifizierung in der Frauenforschung. In Gudrun Knapp-Axeli, \& Angelika Wetterer (Hrsg.), TraditionenBrüche. Entwicklungen feministischer Theorie (S. 201-254). Freiburg: Kore.

Glötzner, Johannes (1982). Heidi häkelt Quadrate, Thomas erklärt die Multiplikation. Rollenklischees in neuen Mathematikbüchern. In Ilse Brehmer (Hrsg.), Sexismus in der Schule. Der heimliche Lehrplan der Frauendiskriminierung. (S. 154-158). Weinheim: Beltz.

Goffman, Erving (2007). Wir alle spielen Theater. Die Selbstdarstellung im Alltag. München: Piper.

Heintz, Bettina, \& Nadai, Eva (1998). Geschlecht und Kontext. DeInstitutionalisierungsprozesse und geschlechtliche Differenzierung. Zeitschrift für Soziologie, 27(2), 75-93.

Hirschauer, Stefan (1994). Die soziale Fortpflanzung der Zweigeschlechtlichkeit. Kölner Zeitschrift für Soziologie und Sozialpsychologie, 46(4), 668-692.

Hirschauer, Stefan (1996). Wie sind Frauen, wie sind Männer? Zweigeschlechtlichkeit als Wissenssystem. In Christiane Eifert, Angelika Epple, Martina Kessel, Marlis Michaelis, Claudia Nowak, Katharina Schicke, \& Dorothea Welteck (Hrsg.), Was sind Frauen? Was sind Männer? Geschlechterkonstruktionen im historischen Wandel (S. 240-256). Frankfurt a.M.: Suhrkamp.

Hirschauer, Stefan (2001). Das Vergessen des Geschlechts. Zur Praxeologie einer Kategorie sozialer Ordnung. In Bettina Heintz (Hrsg.), Geschlechtersoziologie. Kölner Zeitschrift für Soziologie und Sozialpsychologie. (S. 208-235). Wiesbaden: Westdeutscher Verlag.

Hunze, Annette (2003). Geschlechtertypisierung in Schulbüchern. In Monika Stürzer, Henrike Roisch, Annette Hunze, \& Waltraud Cornelissen (Hrsg.), Geschlechterverhältnisse in der Schule DJi-Rehe (Gender), vol 20 (S. 53-81). Opladen: Leske+Budrich.

Kassner, Karsten (2008). Männlichkeitskonstruktionen von 'neuen Vätern'. In Nina Bauer, \& Jens Luedtke (Hrsg.), Die soziale Konstruktion von Männlichkeit. Hegemoniale und marginalisierte Männlichkeiten in Deutschland (S. 141-164). Opladen/Farmington Hills: Barbara Budrich.

Kästner, Sibylle (2012). Jagende Sammlerinnen und sammelnde Jägerinnen. Wie australische Aborigines-Frauen Tiere erbeuten. Berlin: LIT Verlag.

Koppetsch, Cornelia, \& Burkart, Günter (1999). Die Illusion der Emanzipation. Zur Wirksamkeit latenter Geschlechtsnormen im Milieuvergleich. Konstanz: Universitätsverlag Konstanz.

Kraimer, Klaus (2009). Dokumentenanalyse. Studienbrief 8, o. O., S. 1-9.

Lässig, Simone (2010). Wer definiert relevantes Wissen? Schulbücher und ihr gesellschaftlicher Kontext. In Eckhardt Fuchs, Joachim Kahlert, \& Uwe Sandfuchs (Hrsg.). Schulbuch konkret. Kontexte - Produktion - Unterricht (S. 199-215). Bad Heilbrunn: Klinkhardt.

Lenz, Karl, \& Adler, Marina (2010). Geschlechterverhältnisse. Einführung in die sozialwissenschaftliche Geschlechterforschung. Band 1. Weinheim: Juventa. 
MacNaughton, Glenda (2000). Rethinking Gender in Early Childhood Education. London: Paul Chapman.

Markom, Christa, \& Weinhäuptl, Heidi (2007). Die Anderen im Schulbuch. Rassismen, Exotismen, Sexismen und Antisemitismus in österreichischen Schulbüchern. Wien: Braumüller.

Meuser, Michael (2010). Geschlecht und Männlichkeit. Soziologische Theorie und kulturelle Deutungsmuster (3. Auflage) Wiesbaden: VS Verlag für Sozialwissenschaften.

Moser, Franziska, Hannover, Bettina, \& Becker, Judith (2013). Subtile und direkte Mechanismen der sozialen Konstruktion von Geschlecht in Schulbüchern. Vorstellung eines Kategoriensystems zur Analyse der Geschlechter(un)gerechtigkeit von Texten und Bildern. Gender, 5(3), 77-93.

Nentwich, Julia C. (2004). Die Gleichzeitigkeit von Differenz und Gleichheit. Neue Wege für die Gleichstellungsarbeit. Königstein: Ulrike Helmer.

Nentwich, Julia C. (2014). Puppen für die Buben und Autos für die Mädchen? Rhetorische Modernisierung in der Kinderkrippe. In Gerline Malli, \& Susanne Sackl-Shari (Hrsg.), Wider die Gleichheitsrhetorik. Soziologische Analysen - Theoretische Interventionen (S. 50-61). Münster: Westfälisches Dampfboot.

Rabe-Kleberg, U. (2003). Gender Mainstreaming und Kindergarten. Weinheim: Beltz.

Reh, Werner (1995). Quellen- und Dokumentenanalyse in der Politikfeldforschung: Wer steuert die Verkehrspolitik? In Ulrich von Alemann (Hrsg.), Politikwissenschaftliche Methoden. Grundriss für Studium und Forschung (S. 201-259). Wiesbaden: VS Verlag für Sozialwissenschaften.

Rohrmann, Tim, \& Wanzeck-Sielert, Christa (2018). Mädchen und Jungen in der KiTa. Körper, Gender, Sexualität (2., erweiterte und überarbeitete Aufl.). Stuttgart: Kohlhammer.

Rossbach, Hans-Günther, Sechtig, Jutta, \& Schmidt, Thilo (2012). Pädagogik der Frühen Kindheit und Kindertageseinrichtungen. In Werner Thole (Hrsg.), Grundriss Soziale Arbeit. Ein einführendes Handbuch (4. Auflage, S. 461-467). Wiesbaden: VS Verlag für Sozialwissenschaften.

Sandfuchs, Uwe (2010). Schulbücher und Unterrichtsqualität - Historische und aktuelle Reflexionen. In Eckhardt Fuchs, Joachim Kahlert, \& Uwe Sandfuchs (Hrsg.), Schulbuch konkret. Kontexte - Produktion - Unterricht (S. 11-24). Bad Heilbrunn: Klinkhardt.

Schmitz, Sigrid (2006). Jägerinnen und Sammler. Evolutionsgeschichten zur Menschwerdung. In Smilla Ebeling, \& Sigrid Schmitz (Hrsg.), Geschlechterforschung und Naturwissenschaft. Einführung in ein komplexes Wechselspiel (S. 188-210). Wiesbaden: VS Verlag für Sozialwissenschaften.

Soeffner, Hans-Georg (2004). Auslegung des Alltags - Der Alltag der Auslegung. Zur wissenssoziologischen Konzeption einer sozialwissenschaftlichen Hermeneutik. Konstanz: UVK.

Vogt, Franziska, Nentwich, Julia C., \& Tennhoff, Wiebke (2015). Doing und Undoing Gender in Kinderkrippen: Eine Videostudie zu den Interaktionen von Kinderbetreuenden mit Kindern. Schweizerische Zeitschrift für Bildungswissenschaften, 37(2), 227-247.

von Borries, Bodo (1975). Frauen in Schulgeschichtsbüchern. Zum Problem der Benachteiligung von Mädchen im Unterricht. Westermanns pädagogische Beiträge, 27(11), 601-618.

West, Candace, \& Zimmerman, Don H. (2009). Accounting for Doing Gender. Gender \& Society, 23(1), 112-122. 
West, Candace, \& Zimmerman, Don H. (1987). Doing Gender. Gender \& Society, 1(2), 125-151.

Wetterer, Angelika (2003). Rhetorische Modernisierung. Das Verschwinden der Ungleichheit aus dem zeitgenössischen Differenzwissen. In Gudrun Knapp, \& Angelika Wetterer (Hrsg.), Achsen der Differenz. Gesellschaftstheorie und feministische Kritik II. (S. 286-319). Münster: Westfälisches Dampfboot.

Wetterer, Angelika (2008a). Geschlechterwissen. Zur Geschichte eines neuen Begriffs. In Angelika Wetterer (Hrsg.), Geschlechterwissen und soziale Praxis. Theoretische Zugänge - Empirische Erträge (S. 13-36). Königstein im Taunus: Ulrike Helmer.

Wetterer, Angelika (2008b). Geschlechterwissen und soziale Praxis. Grundzüge einer wissenssoziologischen Typologie des Geschlechterwissens. In Angelika Wetterer (Hrsg.), Geschlechterwissen und soziale Praxis. Theoretische Zugänge - Empirische Erträge (S. 39-63). Königstein im Taunus: Ulrike Helmer.

Wiater, Werner (2003). Das Schulbuch als Gegenstand pädagogischer Forschung. In Werner Wiater (Hrsg.), Schulbuchforschung in Europa-Bestandsaufnahme und Zukunftsperspektive (S. 11-21). Bad Heilbrunn: Klinkhardt.

Open Access Dieses Kapitel wird unter der Creative Commons Namensnennung 4.0 International Lizenz (http://creativecommons.org/licenses/by/4.0/deed.de) veröffentlicht, welche die Nutzung, Vervielfältigung, Bearbeitung, Verbreitung und Wiedergabe in jeglichem Medium und Format erlaubt, sofern Sie den/die ursprünglichen Autor(en) und die Quelle ordnungsgemäß nennen, einen Link zur Creative Commons Lizenz beifügen und angeben, ob Änderungen vorgenommen wurden.

Die in diesem Kapitel enthaltenen Bilder und sonstiges Drittmaterial unterliegen ebenfalls der genannten Creative Commons Lizenz, sofern sich aus der Abbildungslegende nichts anderes ergibt. Sofern das betreffende Material nicht unter der genannten Creative Commons Lizenz steht und die betreffende Handlung nicht nach gesetzlichen Vorschriften erlaubt ist, ist für die oben aufgeführten Weiterverwendungen des Materials die Einwilligung des jeweiligen Rechteinhabers einzuholen.

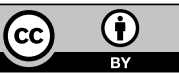

